\title{
Closed-Form Error Analysis of the Non-Identical Nakagami- $m$ Relay Fading Channel
}

\author{
Himal A. Suraweera, Member, IEEE, and George K. Karagiannidis, Senior Member, IEEE
}

\begin{abstract}
We present closed-form expressions for the average bit error probability (ABEP) of BPSK, QPSK and $M$-QAM of an amplify-and-forward average power scaling dual-hop relay transmission, over non-identical Nakagami- $m$ fading channels, with integer values of $m$. Additionally, we evaluate in closedform the ABEP under sufficiently large signal-to-noise ratio for the source-relay link, valid for arbitrary $m$. Numerical and simulation results show the validity of the proposed mathematical analysis and point out the effect of the two hops unbalanced fading conditions on the error performance.
\end{abstract}

Index Terms - Wireless relays, Nakagami- $m$ fading, amplifyand-forward, error performance.

\section{INTRODUCTION}

$\mathbf{I}^{\mathrm{s}}$ $\mathrm{N}$ recent years, wireless relaying techniques have attracted a lot of research interest due to their possible exploit in cellular, ad-hoc networks and military communications [1]. In relay networks, intermediate nodes are used to relay signals between the source and the destination terminal.

Amplify-and-forward (AF) is one of the two main schemes for relaying [2]. AF relays without performing any decoding, retransmit a scaled replica of the received signal. Literature on AF relaying schemes assumes two different power constraints at the relay: fixed-gain [2] also called "average power scaling" (APS) in [3] and instantaneous power scaling [3].

The performance analysis of multihop wireless networks operating under different fading conditions has been an important field of research in the past few years. See for example, [2]-[10]. In [3], Mheidat and Uysal have investigated the impact of receive diversity on the performance of a relayassisted network in which the relay is operating under the AFAPS constraint. In [2], [5], Hasna and Alouini have studied the average bit error probability (ABEP) of dual-hop systems with AF relaying over Rayleigh and Nakagami-m fading channels. In [4], Adinoyi and Yanikomeroglu have analyzed the error performance of a decode-and-forward (DF) based multi-antenna relay network in the presence of Nakagami- $m$ fading. In [6] and [7] Karagiannidis et al. have studied the performance bounds of AF multihop transmissions over nonidentically distributed Nakagami- $m$ fading channels. In [9] Ikki and Ahmed have presented a tight lower bound for the performance of an AF multi-relay network over non-identical

Manuscript received November 16, 2007. The associate editor coordinating the review of this letter and approving it for publication was S. Buzzi.

H. A. Suraweera is with the School of Electrical Engineering, Victoria University, PO Box 14428, Melbourne, Victoria, 8001, Australia (e-mail: himal.suraweera@vu.edu.au).

G. K. Karagiannidis is with the Department of Electrical and Computer Engineering, Aristotle University of Thessaloniki, GR-54124, Greece (e-mail: geokarag@auth.gr).

Digital Object Identifier 10.1109/LCOMM.2008.071922.
Nakagami- $m$ fading channels, especially in the medium and high signal-to-noise (SNR) region.

In this letter, we present closed-form expressions for the ABEP of an AF-APS dual-hop relay link in non-identical Nakagami- $m$ fading channels (which is the real situation in practical wireless relaying systems) with integer fading parameters. To the best of authors' knowledge, no exact closedform ABEP expressions for the non-identical Nakagami-m AF-APS relaying are reported. Moreover, we derive a closedform formula for the error performance under sufficiently large SNR for the source-relay link, valid for arbitrary values of $m$.

\section{DUAL-Hop RElay MODEL}

Consider a wireless communication system, where a source terminal $S$ communicates with a destination terminal $D$ using a relay $R$ [2]. Let the modulated signal transmitted by $S$ during the first time slot denoted as $x$. The received signal at $R$ is given by [2]

$$
y_{r}=\sqrt{E_{S R}} \alpha_{1} x+n_{r}
$$

where $\alpha_{1}$ is the fading amplitude of the $S-R$ link. $n_{r}$ is an additive white Gaussian noise (AWGN) component with single sided power spectral density $N_{0}$. In the second time slot, the relay multiplies the received signal by a gain factor $G$ and then retransmits to $D$. The received signal at $D$ is

$$
y_{d}=\sqrt{E_{R D}} \alpha_{2} G\left(\sqrt{E_{S R}} \alpha_{1} x+n_{r}\right)+n_{d}
$$

where $\alpha_{2}$ is the fading amplitude of the $R-D$ link and $n_{d}$ is the AWGN component with power $N_{0}$ at the input of $D$. $E_{S R}$ and $E_{R D}$ represent the average energies available at $R$ and $D$, taking into consideration of possibly different path loss and shadowing effects in $S-R$ and $R-D$ links [3]. When $R$ operates under APS constraint, $G^{2}=1 /\left(E_{S R}+N_{0}\right)$. The instantaneous end-to-end SNR at $D, \gamma_{\mathrm{eq}}$, is given by [2]

$$
\gamma_{\mathrm{eq}}=\frac{\left(E_{S R} / N_{0}\right)\left(E_{R D} / N_{0}\right) \alpha_{1}^{2} \alpha_{2}^{2}}{1+E_{S R} / N_{0}+\left(E_{R D} / N_{0}\right) \alpha_{2}^{2}}
$$

and (3) can be reexpressed as

$$
\gamma_{\mathrm{eq}}=\frac{\gamma_{1} \gamma_{2}}{C+\gamma_{2}}
$$

where $C=1+\left(E_{S R} / N_{0}\right)$ and $\gamma_{1}=\alpha_{1}^{2} E_{S R} / N_{0}, \gamma_{2}=$ $\alpha_{2}^{2} E_{R D} / N_{0}$ denote the instantaneous SNRs of the $S-R$ and $R-D$ hops respectively. Since the hops are subject to nonidentical Nakagami fading, we model $\alpha_{1}$ and $\alpha_{2}$ according to Nakagami- $m$ distribution with fading severity parameters $m_{1}$ and $m_{2}$, respectively, i.e.,

$$
p_{\alpha_{i}}(\alpha)=\frac{2 m_{i}^{m_{i}} \alpha^{2 m_{i}-1} e^{-m_{i} \alpha^{2}}}{\Gamma\left(m_{i}\right)}
$$


where $i=1,2$ and $\Gamma(z)=\int_{0}^{\infty} t^{z-1} e^{-t} d t$ is the gamma function. In the probability density functions (pdfs) of $\alpha_{1}$ and $\alpha_{2}$, without loss of generality, we have set $E\left\{\alpha_{1}^{2}\right\}$ and $E\left\{\alpha_{2}^{2}\right\}$ to unity. Since $\alpha_{i}$ is modeled as a Nakagami-m random variable (RV), the instantaneous SNR $\gamma_{i}$ is a gamma distributed RV with pdf given by

$$
p_{\gamma_{i}}(\gamma)=\frac{m_{i}^{m_{i}} \gamma^{m_{i}-1} e^{-m_{i} \gamma / \Omega_{i}}}{\Omega_{i}^{m_{i}} \Gamma\left(m_{i}\right)}
$$

where $\Omega_{1}=E_{S R} / N_{0}$ and $\Omega_{2}=E_{R D} / N_{0}$.

\section{ERROR ANALYSIS}

Traditionally the ABEP is computed by determining the pdf of $\gamma_{\mathrm{eq}}$ and then averaging the conditional BEP in AWGN, $P_{b}(e \mid \gamma)$, over this pdf. Mathematically, $P_{b}(e)$ is given by

$$
P_{b}(e)=\int_{0}^{\infty} p(e \mid \gamma) p_{\gamma_{\mathrm{eq}}}(\gamma) d \gamma
$$

Note that for several Gray bit-mapped constellations employed in practical systems, $P_{b}(e \mid \gamma)$ is in the form of $Q(\sqrt{\beta \gamma})$ with $Q(x)$ being the Gaussian $Q$-function defined as $Q(x)=$ $(1 / \sqrt{2 \pi}) \int_{x}^{\infty} e^{-t^{2} / 2} d t$ and $\beta$ is a constant (BPSK: $P_{b}(e \mid \gamma)=$ $Q(\sqrt{2 \gamma})$, QPSK: $P_{b}(e \mid \gamma)=Q(\sqrt{\gamma})$ and in the case of square/rectangular $M$-QAM, $P_{b}(e \mid \gamma)$ can be written as a finite weighted sum of $Q(\sqrt{\beta \gamma})$ terms [11]).

To evaluate the integral in (7), we invoke the technique described in [10]. That is, after introducing a new RV with standard Normal distribution, $P_{b}(e)=\int_{0}^{\infty} Q(\sqrt{\beta \gamma}) p_{\gamma_{\mathrm{eq}}}(\gamma) d \gamma$ can be reexpressed as

$$
P_{b}(e)=\frac{1}{\sqrt{2 \pi}} \int_{0}^{\infty} F_{\gamma_{\mathrm{eq}}}\left(t^{2} / \beta\right) e^{-t^{2} / 2} d t
$$

Fortunately, Tsiftsis et al. in [8] derived the cumulative distribution function of $\gamma_{\mathrm{eq}}, F_{\gamma_{\mathrm{eq}}}(\gamma)$, valid for integer $m_{1}$ and $m_{2}$. Using [8, eq. 18] $F_{\gamma_{\text {eq }}}(\gamma)$ can be written as ${ }^{1}$

$$
\begin{aligned}
F_{\gamma_{\mathrm{eq}}}(\gamma)=1-\sum_{i=0}^{m_{1}-1} \sum_{j=0}^{i} \Upsilon(i, j) e^{-m_{1} \gamma / \Omega_{1}} \\
\gamma^{\frac{2 i+m_{2}-j}{2}} K_{m_{2}-j}\left(2 \sqrt{\frac{m_{1} m_{2} C \gamma}{\Omega_{1} \Omega_{2}}}\right)
\end{aligned}
$$

and

$$
\Upsilon(i, j)=\frac{2\left(\begin{array}{l}
i \\
j
\end{array}\right)}{\Gamma\left(m_{2}\right) i !}\left(\frac{m_{1}}{\Omega_{1}}\right)^{\frac{2 i+m_{2}-j}{2}}\left(\frac{C m_{2}}{\Omega_{2}}\right)^{\frac{m_{2}+j}{2}}
$$

In (9) $K_{\nu}(\cdot)$ is the $\nu$-th order modified Bessel function of the second kind. Substituting (9) into (8) and using [12, eq. 2.16.8.4] $P_{b}(e)$ can be computed in closed-form as

$$
\begin{aligned}
& P_{b}(e)=\frac{1}{2}-\frac{1}{\sqrt{2 \pi}} \sum_{i=0}^{m_{1}-1} \sum_{j=0}^{i} \frac{0.25 \Upsilon(i, j) \vartheta^{\lambda_{1}} \Gamma\left(i+\frac{1}{2}\right)}{\left(\beta\left(\frac{1}{2}+\frac{m_{1}}{\Omega_{1} \beta}\right)\right)^{\lambda_{2}}} \\
& \cdot \frac{1}{\left(\frac{m_{1} m_{2} C}{\Omega_{1} \Omega_{2} \beta}\right)^{\frac{1}{2}}} \Gamma\left(2 \lambda_{2}-i+\frac{1}{2}\right) \Psi\left(2 \lambda_{2}-i+\frac{1}{2}, 2 \lambda_{1} ; \vartheta\right)
\end{aligned}
$$

${ }^{1}$ It is noted that Eqs. 18 and 19 in [8] include typos which we have corrected in (9) and (10). where $\lambda_{1}=\frac{m_{2}-j+1}{2}, \lambda_{2}=\frac{2 i+m_{2}-j}{2}, \vartheta=\frac{2 m_{1} m_{2} C}{\left(2 m_{1}+\Omega_{1} \beta\right) \Omega_{2}}$ and $\Psi(a, b ; z)$ is the Tricomi confluent hypergeometric function $[13$, p. 504]. Note that to arrive at (11) we have employed the well known relationship between the Whittaker function and $\Psi(a, b ; z)[13$, p. 505].

In the special case of Rayleigh fading, a closed-form for $P_{b}(e)$ can be obtained setting $m_{1}=m_{2}=1$, in (11) and after some manipulations as

$$
\begin{aligned}
P_{b}(e) & =\frac{1}{2}-\xi \int_{0}^{\infty} t e^{-\left(\frac{2+\Omega_{1}}{2 \Omega_{1}}\right) t^{2}} K_{1}\left(2 \sqrt{\frac{C}{\Omega_{1} \Omega_{2}}} t\right) d t \\
& =\frac{1}{2}\left(1-\frac{\ell}{\sqrt{1+\left(2 / \Omega_{1} \beta\right)}} e^{\ell}\left[K_{1}(\ell)-K_{0}(\ell)\right]\right)
\end{aligned}
$$

where $\xi=\sqrt{\frac{2 C}{\pi \Omega_{1} \Omega_{2} \beta}}$ and $\ell=\frac{C}{\left(2+\Omega_{1} \beta\right) \Omega_{2}}$. Note, that (12) can be also derived from (7) and [2, eq. 9], pointing out the validity and the generality of our approach.

\section{A. ABEP under Sufficiently Large SNR for $S$ - $R$ Link and Arbitrary $m$}

In order to derive the ABEP for arbitrary $m$, we assume sufficiently large SNR for the $S-R$ hop [3], i.e., $E_{S R} / N_{0}>$ $E_{R D} / N_{0}$. Under this assumption, the end-to-end SNR is [3]

$$
\omega_{\mathrm{eq}}=\frac{E_{R D}}{N_{0}} \alpha_{1}^{2} \alpha_{2}^{2}
$$

A squared Nakagami RV is gamma distributed. Let two independent RVs $X$ and $Y$ be gamma distributed, i.e., $X \sim$ $\mathcal{G}\left(a_{X}, b_{X}\right)$ and $Y \sim \mathcal{G}\left(a_{Y}, b_{Y}\right)$. The pdf of the product $Z$ of $X$ and $Y$ is given by

$$
\begin{aligned}
p_{Z}(z) & =\int_{0}^{\infty} \frac{1}{t} p_{X}(t) p_{Y}\left(\frac{z}{t}\right) d t \\
& =\frac{2}{\Gamma\left(a_{X}\right) \Gamma\left(a_{Y}\right)}\left(b_{X} b_{Y}\right)^{-\frac{a_{X}+a_{Y}}{2}} z^{\left(\frac{a_{X}+a_{Y}}{2}-1\right)} \\
& \times K_{a_{Y}-a_{X}}\left(2 \sqrt{\frac{z}{b_{X} b_{Y}}}\right)
\end{aligned}
$$

Therefore, the pdf of $\omega_{\text {eq }}$ can be expressed as

$$
\begin{aligned}
p_{\omega_{\mathrm{eq}}}(\omega)=\frac{2}{\Gamma\left(m_{1}\right) \Gamma\left(m_{2}\right)} & \left(\frac{m_{1} m_{2}}{\Omega_{2}}\right)^{\frac{m_{1}+m_{2}}{2}} \omega^{\frac{m_{1}+m_{2}}{2}-1} \\
& \times K_{m_{2}-m_{1}}\left(2 \sqrt{\frac{m_{1} m_{2}}{\Omega_{2}} \omega}\right)
\end{aligned}
$$

Making the substitution $t^{2}=\omega$ and using the formula $Q(x)=$ $0.5 \operatorname{erfc}(x / \sqrt{2})$, the ABEP is given by

$$
\begin{aligned}
P_{b}(e) & =\frac{2\left(\frac{m_{1} m_{2}}{\Omega_{2}}\right)^{\frac{m_{1}+m_{2}}{2}}}{\Gamma\left(m_{1}\right) \Gamma\left(m_{2}\right)} \int_{0}^{\infty} t^{m_{1}+m_{2}-1} \\
& \times \operatorname{erfc}\left(\sqrt{\frac{\beta}{2}} t\right) K_{m_{2}-m_{1}}\left(2 \sqrt{\frac{m_{1} m_{2}}{\Omega_{2}}} t\right)
\end{aligned}
$$

The involved integral in (16) can be evaluated using [12, 2.16.59.1] and the ABEP is expressed as shown in (17) where ${ }_{2} F_{2}(a, b ; c, d ; z)$ is a generalized hypergeometric function [12]. Note that $\Psi(\cdot, \cdot ; z)$ and ${ }_{2} F_{2}(a, b ; c, d ; z)$ can be evaluated using popular symbolic software such as MAPLE, MATHEMATICA and MATLAB. 


$$
\begin{aligned}
P_{b}(e)=\frac{1}{\sqrt{\pi} \Gamma\left(m_{1}\right) \Gamma\left(m_{2}\right)}\left[\frac{2^{m_{1}-1} \Gamma\left(m_{2}-m_{1}\right) \Gamma\left(0.5+m_{1}\right)}{m_{1}\left(m_{1} m_{2} / \Omega_{2} \beta\right)^{-m_{1}}}{ }_{2} F_{2}\left(m_{1}, 0.5+m_{1} ; 1+m_{1}, 1+m_{1}-m_{2} ; \frac{2 m_{1} m_{2}}{\Omega_{2} \beta}\right)\right. \\
\left.+\frac{2^{m_{2}-1} \Gamma\left(m_{1}-m_{2}\right) \Gamma\left(0.5+m_{2}\right)}{m_{2}\left(m_{1} m_{2} / \Omega_{2} \beta\right)^{-m_{2}}}{ }_{2} F_{2}\left(m_{2}, 0.5+m_{2} ; 1+m_{2}, 1+m_{2}-m_{1} ; \frac{2 m_{1} m_{2}}{\Omega_{2} \beta}\right)\right]
\end{aligned}
$$

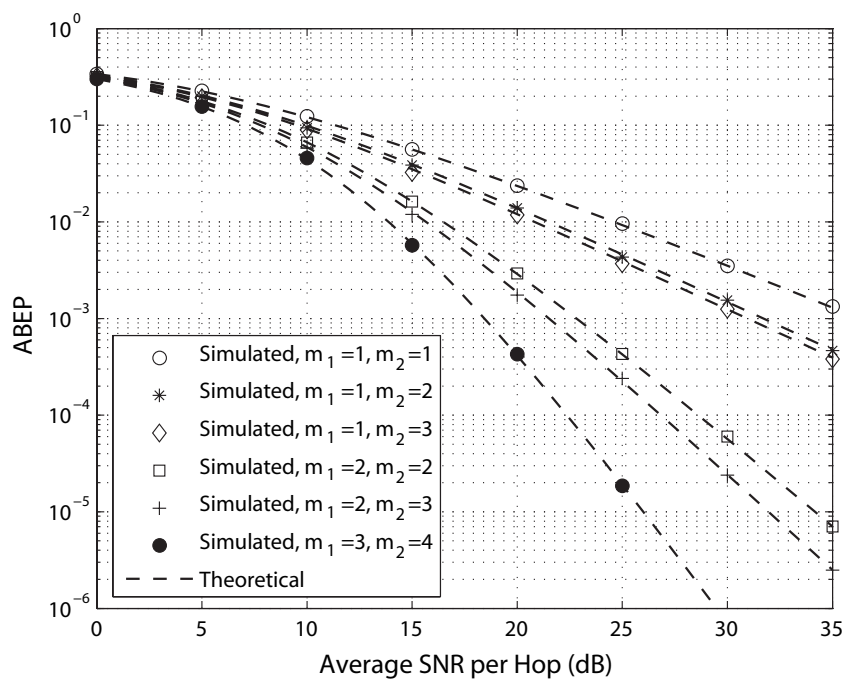

Fig. 1. Simulated and theoretical ABEP of the dual-hop relay link.

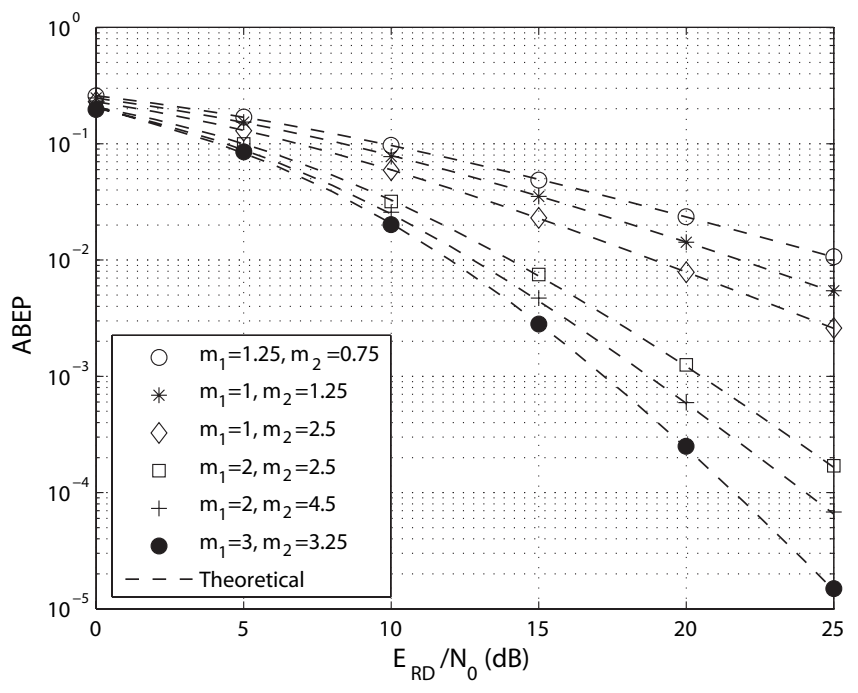

Fig. 2. Comparison of simulated and theoretical ABEP. $\Omega_{1}=35 \mathrm{~dB}$.

\section{NumericAl AND Simulation Results}

Fig. 1 shows the ABEP of 4-QAM against the average SNR per hop, different values of $m$ and $E_{S R} / N_{0}=E_{R D} / N_{0}$. It should be noted, that although AF relaying will decrease the complexity at the relay, the destination needs to have channel state information knowledge of both the $S-R$ and the $R-D$ links. For comparison, the Rayleigh faded relay performance is also plotted. Observe that all the numerical results (the curves) are in exact agreement with the simulated ABEP results. With $S-R$ link subject to Rayleigh fading, no significant improvement in ABEP can be obtained for $m>3$ in the $R$ $D$ link. However, improved fading severity conditions in both links lower the ABEP significantly and the achieved diversity order is increased. As noticed from Fig. 1 for an ABEP equal to $10^{-5}$, when fading severity changes from $m_{1}=m_{2}=2$ to $m_{1}=3, m_{2}=4$, a SNR gain of $8 \mathrm{~dB}$ can be achieved. Fig. 2 illustrates the ABEP of the dual-hop relay link when the average SNR of the $S-R$ link is $35 \mathrm{~dB}$ [3]. Again as expected, the error performance improves for large $m$ values in both hops. Finally, simulations were performed to check the validity of (17). As it is evident from Fig. 2 they perfectly match with the analytical ABEP.

\section{CONCLUSion}

We have derived closed-form ABEP expressions for several modulation schemes of an AF-APS dual-hop relay link operating in independent and non-identical Nakagami- $m$ fading channels. This analysis is useful to investigate the performance of AF-APS relaying subject to different fading conditions both for source to relay and relay to destination links.

\section{REFERENCES}

[1] R. Pabst et al., "Relay-based deployment concepts for wireless and mobile broadband radio," IEEE Commun. Mag., vol. 42, pp. 80-89, Sept. 2004.

[2] M. O. Hasna and M.-S. Alouini, "A performance study of dual-hop transmissions with fixed gain relays," IEEE Trans. Wireless Commun., vol. 3, pp. 1963-1968, Nov. 2004.

[3] H. Mheidat and M. Uysal, "Impact of receive diversity on the performance of amplify-and-forward relaying under APS and IPS power constraints," IEEE Commun. Lett., vol. 10, pp. 468-470, June 2006.

[4] A. Adinoyi and H. Yanikomeroglu, "Cooperative relaying in multiantenna fixed relay networks," IEEE Trans. Wireless Commun., vol. 6, pp. 533-544, Feb. 2007

[5] M. O. Hasna and M. S. Alouini, "Harmonic mean and end-to-end performance of transmission systems with relays," IEEE Trans. Commun., vol. 52, pp. 130-135, Jan. 2004.

[6] G. K. Karagiannidis, T. A. Tsiftsis, and R. K. Mallik, "Bounds of multihop relayed communications in Nakagami- $m$ fading," IEEE Trans. Commun., vol. 54, pp. 18-22, Jan. 2006.

[7] G. K. Karagiannidis, "Performance bounds of multihop wireless communications with blind relays over generalized fading channels," IEEE Trans. Wireless Commun., vol. 5, pp. 498-503, Mar. 2006.

[8] T. A. Tsiftsis, G. K. Karagiannidis, P. T. Mathiopoulos, and S. A. Kotsopoulos, "Nonregenerative dual-hop cooperative links with selection diversity," EURASIP J. Wireless Commun. Networking, vol. 2006, article ID 17862, eight pages.

[9] S. Ikki and M. H. Ahmed, "Performance analysis of cooperative diversity wireless networks over Nakagami- $m$ channels," IEEE Commun. Lett., vol. 11, pp. 334-336, Apr. 2007.

[10] Y. Zhao, R. Adve, and T. J. Lim, "Symbol error rate of selection amplifyand-forward relay systems," IEEE Commun. Lett., vol. 10, pp. 757-759, Nov. 2006.

[11] K. Cho and D. Yoon, "On the general BER expression of one and two dimensional amplitude modulations," IEEE Trans. Commun., vol. 50, pp. 1074-1080, July 2002.

[12] A. P. Prudnikov, Y. A. Brychkov, and O. I. Marichev, Integrals and Series, vol. 2. Gordon and Breach Science Publishers, 1986.

[13] M. Abramowitz and I. A. Stegun, Handbook of Mathematical Functions with Formulas, Graphs, and Mathematical Tables, 10th ed. New York: Dover, 1972. 